\title{
Semantic validation of the ISPCAN Child Abuse Screening Tools (ICAST) in Brazilian Portuguese
}

\author{
Validação semântica da ISPCAN Child Abuse Screening Tools (ICAST) em \\ português brasileiro
}

Ana Ligia da Silva Silveira, Rodrigo Grassi-Oliveira*

\begin{abstract}
Objective: The International Society for the Prevention of Child Abuse and Neglect (ISPCAN) Child Abuse Screening Tools (ICAST) is a battery of questionnaires created by the World Health Organization, the United Nations and ISPCAN for researching maltreatment in childhood. This study aims to translate, to adapt and to validate the semantic equivalence of all items on the three questionnaires: ICAST-C (ICAST version for Children), ICAST-R (Retrospective Interview) and ICAST-P (ICAST version for Parents).

Methods: The process of translation and semantic validation comprised five methodological steps: 1) translation; 2) back-translation; 3) correction and semantic adaptation; 4) validation of content by professional experts in the area of abuse in childhood; and 5) a study of their acceptability to a sample of the target population, using a verbal rating scale.

Results: In the evaluation of the expert committee, there was need to adapt several words for the Brazilian population while maintaining semantic and conceptual equivalence. In the ICAST-C acceptability study, children exhibited some difficulty understanding 7 of the items (out of 69 questions). For ICAST-P, parents reported a lack of clarity in 5 items (out of 57 questions). These issues were resolved and the Brazilian version of ICAST 3.0 was concluded.

Conclusion: The ICAST battery is an internationally recognized tool and the process of translation into Portuguese and semantic adaptation was performed successfully. The final version proved to be easily understandable and semantic validation results were adequate. This battery has proved useful in investigation of childhood maltreatment.
\end{abstract}

Keywords: Child abuse, assessment, trauma, stress, instrument, childhood, maltreatment, neglect, interview.

\section{Resumo}

Objetivo: A Child Abuse Screening Tools (ICAST), da International Society for the Prevention of Child Abuse and Neglect (ISPCAN), é uma bateria de questionários criada pela Organização Mundial da Saúde, Nações Unidas e ISPCAN para a investigação de maus-tratos na infância. Este estudo visa traduzir, adaptar e realizar a validação semântica de todos os itens dos três questionários: ICAST-C (ICAST versão para Crianças), ICAST-R (Entrevista Retrospectiva) e ICAST-P (ICAST versão para Pais).

Métodos: O processo de tradução e validação semântica incluiu cinco etapas metodológicas: 1) tradução; 2) retrotradução; 3) correção e adaptação semântica; 4) validação de conteúdo por profissionais especialistas na área de maus-tratos na infância; e 5) um estudo de aceitabilidade por uma amostra da população-alvo, usando uma escala de graduação verbal.

Resultados: Na avaliação pelo comitê de especialistas, houve a necessidade de adaptação de muitas palavras para a população brasileira, mantendo a equivalência conceitual e semântica. No estudo de aceitabilidade do ICAST-C, as crianças mostraram alguma dificuldade na compreensão de 7 itens (de um total de 69 questões). No ICAST-P, os pais julgaram que 5 itens não estavam claros (de um total de 57 questões). Essas dificuldades foram corrigidas e a versão brasileira do ICAST 3.0 foi concluída.

Conclusões: A bateria ICAST é uma ferramenta internacionalmente reconhecida, e o processo de tradução para o português e sua adaptação semântica foram realizados com sucesso. A versão final mostrou ser de fácil entendimento, e os resultados da validação semântica foram adequados. Essa bateria demonstrou ser útil na investigação de maus-tratos na infância.

Descritores: Abuso na infância, avaliação trauma, estresse, instrumento, infância, maus-tratos, negligência, entrevista.

\footnotetext{
* Developmental Cognitive Neuroscience Lab (DCNL), Pontifícia Universidade Católica do Rio Grande do Sul (PUCRS), Porto Alegre, RS, Brazil. Financial support: none.

Submitted Dec 02 2015, accepted for publication Feb 05 2016. No conflicts of interest declared concerning the publication of this article.

Suggested citation: Silveira AL, Grassi-Oliveira R. Semantic validation of the ISPCAN Child Abuse Screening Tools (ICAST) in Brazilian Portuguese. Trends Psychiatry Psychother. 2016;38(2):105-110. http://dx.doi.org/10.1590/2237-6089-2016-0012
} 


\section{Introduction}

Over the last 20 years, violence against children and adolescents has become more evident internationally and sexual, physical, and psychological abuse, as well as physical or emotional neglect, have been described as strong social and environmental risk factors for many of the leading causes of death, disease, and disability during all stages of life. ${ }^{1,2}$ Specifically, a recent metaanalysis suggested that childhood abuse and neglect are more frequent in less developed countries and that cultural and economic factors can strongly influence such rates. ${ }^{3}$ In Brazil specifically, there is a paucity of epidemiological data on the extent of violence: there is lack of statistical data, child abuse is underreported and many people see physical punishment as an acceptable method of disciplining children. ${ }^{4}$ Despite the existence of systems for notifiable/reportable events, many cases of child abuse go undetected. Some studies have attempted to identify the causes of this complex issue and found underreporting by health professionals and society. ${ }^{5}$ Another limiting factor is that few childhood maltreatment assessment tools adapted for Brazilian Portuguese are available. ${ }^{6,7}$

In this regard, tools used to investigate child abuse must address the main types of violence with sensitivity and accuracy, ${ }^{7}$ including physical, sexual, or emotional abuse and physical or emotional neglect. ${ }^{8}$ Given the importance of this, in fulfillment of recommendations made in 2001 by the United Nations Commission on the Rights of the Child, the United Nations General Assembly passed Resolution 56/138 calling for a consensus between countries on protection of children and the creation of a new research tool. The International Society for the Prevention of Child Abuse and Neglect (ISPCAN) together with the United Nations Children's Fund (UNICEF) agreed to fulfill the following recommendation: collect data on child abuse and assist in the development of standardized instruments to assess child maltreatment. ${ }^{9}$

The ISPCAN is a non-governmental organization that investigates child abuse and is attached to the University of North Carolina. In 2009, the ISPCAN and UNESCO gathered a group of scientists from 40 countries for the purpose of developing questionnaires on violence against children. These tools were revised by more than 100 experts from different countries and the Delphi process was used to arrive at a final versions, which were named the ISPCAN - Child Abuse Screening Tools (ICAST). ${ }^{10}$

Version 1.0 of the ICAST comprised four questionnaires. There were two versions of the ISPCAN Child Abuse Screening Tool - Children's version (ICAST-C), a home version and an institutional (school) version. The aim was to develop an easy-to-read and self-administered tool that addressed the four types of child abuse. The questions concerned situations of violence occurred in the previous year. As part of development, 571 students aged 12-18 years were recruited for a pilot study in four countries (Colombia, India, Russia, and Iceland). ${ }^{11}$ The scale was translated into Spanish, Hindi, Russian, and Icelandic. In addition to the children's version, a retrospective interview was created for young adults (ICAST-R). The pilot study for this instrument involved 842 participants aged 18-26 years and was conducted in Colombia, Egypt, India, Kyrgyzstan, Lebanon, Malaysia, and Russia, and the questionnaire was translated into these countries' respective languages. The questions addressed physical, emotional and sexual events, questions about the perpetrator, characteristics and frequency of the acts and when each event occurred during childhood. ${ }^{12}$ The fourth questionnaire was called the ICAST-P and was the parent/caregiver version. Parents with children under 18 years old were invited to participate. The questions posed by the researchers were related to the regions in which the pilot study participants lived. These questions concerned parental behaviors, common forms of discipline in their countries and any form of abuse perceived by researchers. In their revision of the questionnaires, the judges selected common issues related to disciplinary practices adopted by these parents in the previous year. ${ }^{13}$

The first versions of the four questionnaires have been thoroughly revised, including standardization of response options in terms of time and frequency of exposure. Response options are presented in descending order (from the highest to the lowest rate of occurrence), as in medical interviews when a physician asks a patient about their rate of drinking. This order of answer choices would cause patients to reveal low drinking rates because they are less serious. The authors conclude that, despite the effort to moderate the risk of intense psychological distress during questions and the very low risks in similar approaches, ${ }^{13}$ these tools should only be administrated by experts in countries that adopt policies of child protection. All these changes were tested in a pilot study and, after an intermediate preliminary version, ICAST 3.0 was completed, which is the latest version developed. This version comprises 3 questionnaires: the ICAST-C (ICAST Children's version), the ICAST-R (Retrospective Interview) and the ICAST-P (ICAST Parent Version). ${ }^{14}$

Version 3.0 of ICAST is a battery of questionnaires designed to standardize the assessment of situations of violence against children and adolescents internationally. ${ }^{14}$ This version has already been used in more than 20 countries, providing knowledge and comparing child abuse in several cultures. Given 
the importance of ICAST tools for data collection and standardization of information in several countries, the present study aims to translate and adapt into Brazilian Portuguese all items on all three ICAST questionnaires, (ICAST-C, ICAST-R, and ICAST-P) and validate their semantic equivalence of.

\section{Methods}

\section{ICAST}

The ICAST-C consists of 69 items and was designed for children and adolescents aged $11-18$ years. Since child abuse often occurs as a private practice, the questions concern the children's experiences in their family environment. This instrument investigates the relationships of respondents with their parents and/or caregivers with relation to discipline (punishments) and to physical or verbal abuse, deprivation and exposure to sexual abuse. According to its authors, asking direct questions about situations of violence provides a better understanding of the issue. The questionnaire has high internal consistency for questions on exposure to violence (Cronbach's alpha 0.69), physical abuse (Cronbach's alpha 0.77), emotional abuse (Cronbach's alpha 0.78), sexual abuse (Cronbach's alpha 0.72) and neglect (Cronbach's alpha 0.83) in the home environment and also for exposure to physical violence (Cronbach's alpha 0.85 ), emotional violence (Cronbach's alpha 0.86) and sexual violence (Cronbach's alpha 0.78) in the school/ work context. However, the authors recognize limitations related to estimation of the real rates of violence in each country. Additionally, their samples were small and the participants were schoolchildren. ${ }^{11}$
The ICAST-P is administered to parents or caregivers and consists of 57 items, including questions on the relationship of the respondent with the target-child. The tool contains questions on the methods used by parents and/or caregivers to discipline the child or adolescent, as well as the attitudes adopted to solve behavioral problems and the frequency of omissions and/or aggressions by respondents. The internal consistency was adequate for questions on non-violent disciplinary practices (Cronbach's alpha 0.77), violent disciplinary practices (Cronbach's alpha 0.88) and emotional abuse (Cronbach's alpha 0.77). However, it was unsatisfactory for sexual abuse (Cronbach's alpha 0.38) and neglect (Cronbach's alpha 0.38). ${ }^{13}$

The ICAST-R was designed for use in young adults. It is composed of 32 items and the questions concern experiences of situations of violence that occurred during childhood up to 18 years of age. It investigates data related to assaults, insults, and threat of abandonment, death, neglect, and sexual abuse. The random sample included university students, street workers, and users of health clinics. The questionnaire has high internal consistency for questions on sexual abuse (Cronbach's alpha 0.86) and moderate internal consistency for physical abuse (Cronbach's alpha 0.60) and emotional abuse (Cronbach's alpha 0.62). ${ }^{12}$

Administration of the ICAST questionnaires begins with an explanation of the purposes of the questions. The participant is guided in self-administered items. The questions concern events that happened during the previous year, except for the young adult version. Interviewers must be trained to administer the questionnaire. Secrecy is ensured and guidance is provided concerning referral to psychology services when required. All questionnaires address socio-demographic data such as gender, age, education, religion, ethnicity,

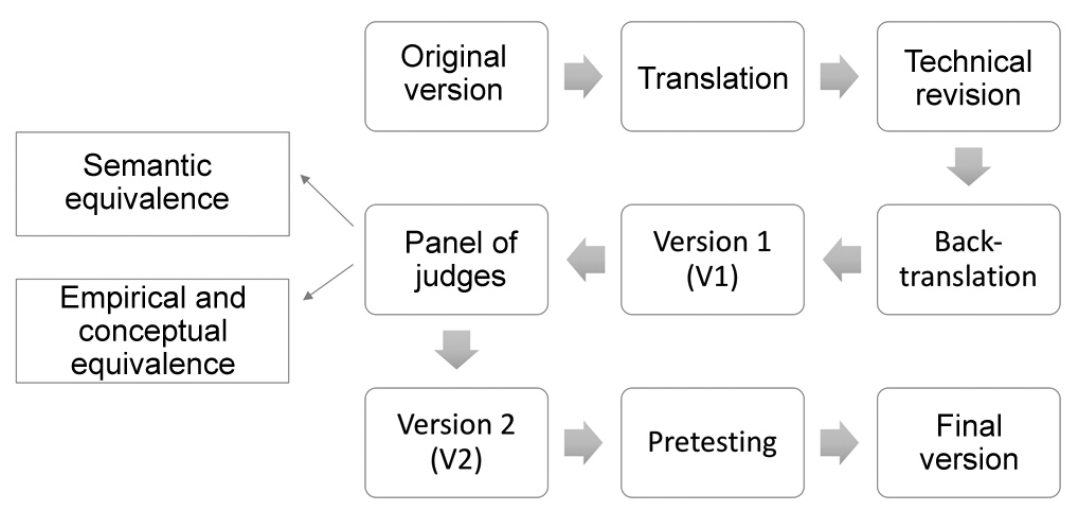

Figure 1 - Flowchart: translation and cross-cultural adaptation for Brazil of the Child Abuse Screening Tools (ICAST). 
people who live with the respondent, place of residence and work activity.

\section{Translation and adaptation}

The procedures for translation and adaptation of the ICAST instrument observed recommended international standards.15,16 A flowchart illustrating the ICAST adaptation process is shown in Figure 1 and this adaptation was performed with prior authorization from ISPCAN.

The first step consisted of translation ( $T$ ) of the original English questionnaire into Brazilian Portuguese, which was performed by two bilingual translators, with degrees in Linguistics, with a major in English and familiar with the subject of violence against children, who produced two independent translations. In the second step, a bilingual translator whose native language is English assessed these two translations and backtranslated them within one document. The third step consisted of a technical review in which each item of the original English version was compared to the backtranslation. Each step was independently performed by two bilingual health care professionals familiar with design and adaptation of instruments and testing in the area of psychology and child abuse. Emphasis was given to semantic equivalence between the words used in the original instrument, the translations and the backtranslation. ${ }^{17}$ This process was performed for each of the three questionnaires and so one preliminary version (V1) was prepared of each questionnaire.

In the fourth step, V1 was submitted to a panel of judges formed by seven experts in mental health who were familiar with the subject of child abuse (two psychiatrists, four psychologists and one child neurologist). These judges compared the items in the original instruments with those in their respective V1s and suggested changes if they detected discrepancies in meaning. The suggestions made by this panel were based on four aspects: semantic equivalence, idiomatic equivalence, empirical equivalence and conceptual equivalence. ${ }^{15,16}$ The review of semantic equivalence aims to assess the similarity of meaning of each item in the original and translated versions. Idiomatic equivalence means that the equivalent expressions should be formulated in the target-version in such a manner as to avoid difficulties caused by translation of colloquialisms and idioms. The review of empirical equivalence aims to identify words in the original questionnaire that should be replaced by similar words that are more consistent with the target culture. Finally, the review of conceptual equivalence checks for words with different cultural meanings and replaces inappropriate words.
Based on the suggestions made, the questionnaires were adapted and a second version (V2) was produced. In the fifth step, V2 versions were shown to target population samples, with the objective of assessing the adequacy of the questions and to check whether they could be understood by each population. The second version (V2) of each instrument was presented to 15 participants recruited in the pediatric outpatients sector of a public general hospital and selected by convenience sampling according to the age range of the target-population to include five participants for each instrument. Thus, patients, parents of patients and workers at the pediatric outpatients unit of a public hospital were invited to participate in the study. Therefore, we included five children aged 10-14 years who had completed basic education to provide inputs about the ICAST-C, five adults from 18-24 years old with educational levels ranging from complete high school to incomplete higher education to comment on the ICAST-R, and five parents of patients who had children of school age and were aged 24 to 52 and had educational levels ranging from complete high school to postgraduate level to give their feedback on the ICAST-P.

A 5-point verbal-numerical scale was used by the participants to rate their understanding of each of the questions in the instruments. The guiding question was: "Did you understand what was asked?" The minimum value was 0 ("I did not understand anything") and the maximum value was 5 ("I have understood perfectly and have no doubts"). The scores $0,1,2$, and 3 were considered indicators of insufficient understanding and scores 4 and 5 indicated sufficient understanding. ${ }^{17}$ Subsequently, the authors analyzed the answers. During this step, a third version (V3) of each instrument was generated including corrections to items that might pose some difficulties for respondents.

All participants signed an informed consent form, as did parents/caregivers of minors, and the institutional ethics committee approved this study.

\section{Results}

The process adopted in the translation and adaptation observed the international standards recommended by Guillemin et al. ${ }^{16}$ In general, the steps of translation and back-translation exhibited great similarity to the original version. During the evaluation by the panel of judges, some words had to be adapted for the Brazilian culture, though maintaining semantic, conceptual, empirical and idiomatic equivalence. Some words were replaced by others, as follows: In ICAST-C, the word "mulher" was replaced by "menina," "homem" was replaced by "menino," "etnia" was 
replaced by "raça," "insultou" was replaced by "xingou," "chacoalhou" was replaced by "sacudiu," "deficiência" was replaced by "atraso," "invocou" was replaced by "chamou," "tutores" was replaced by "cuidadores," "amaldiçoar" was replaced by "rogar pragas," "obsceno" was replaced by "conteúdo sexual," and "álcool" was replaced by "bebida alcoólica." Replacements were also made in some questions related to dangerous situations. For example, "explosão de bombas" was replaced by "tiroteio" in order to respect the cultural adaptation (there are no bombing events in Brazil, but there are many shooting episodes).

Responses were only assessed for degree of understanding of the questionnaire. There was no intention to investigate abuse. The results obtained in pretesting indicated that the questions on the three ICAST instruments were understandable to the respondents. In ICAST-R, with 33 items, value 5 on the verbal-numerical scale predominated (I have perfectly understood and have no doubts). In ICAST-P, comprising 57 items, questions D12, D14, D18, D25, 05 and 06 were scored 3 by one participant. In ICAST-C, comprising 69 questions, a score of 3 was allocated to questions B5 (two participants), B10 (one participant), B11 (one participant), D9a (one participant), D25a (two participants) D27a (one participant) and D29a (one participant).

In general, the mean scores for understanding on the verbal-numerical scale for instrument ICAST-R (mean [M] = 4.84; standard deviation $[\mathrm{SD}]=0.21)$, ICAST-P $(\mathrm{M}=$ 4.69; SD = 0.47), ICAST-C (M=4.45; SD = 0.55) showed that the three instruments obtained an average degree of understanding of 4 on the verbal-numerical scale, indicating a satisfactory degree of understanding. Since the instrument is comprehensive, all questionnaires are available online as supplementary material.

\section{Discussion and conclusion}

Investigating the history of violence in any given population involves addressing highly sensitive issues. Since these issues may cause embarrassment, self-blame and fear, studies that ensure the anonymity of participants and do not make moral judgments about them generate more reliable data and are likely to benefit these individuals. ${ }^{6}$ Therefore, studies based on instruments that consider the cultural context and do not expose the participants are more appropriate and reliable.

The ICAST 3.0 battery is composed of easy-tounderstand questionnaires that have been adopted in several different countries and which facilitate collection and comparison of child abuse data across several countries. ${ }^{14}$ The process of translation and semantic validation to
Brazilian Portuguese was successfully performed in five methodological steps.

In addition to the process of translation and backtranslation of the original instrument, semantic validation was performed with the collaboration of experts in the area and members of the target-population. Without this collaboration, the adaptation of instruments is less significant and more likely to restrain the instrument to its objective meaning. Since most questionnaires are developed in English-speaking countries, use of such instruments in a different country, culture or language requires specific methods to ensure equivalence between the original instrument and the translated version.

The ICAST battery has been validated in more than 20 different languages, it has reproducible properties, allowing for systematic comparison of data across cultures, times or research groups. In this regard, this study has successfully created a Portuguese Brazilian version of the ICAST.

\section{References}

1. Hughes K, Lowey $\mathrm{H}$, Quigg Z, Bellis MA. Relationships between adverse childhood experiences and adult mental well-being: results from an English national household survey. BMC Public Health. 2016;16:222.

2. Bellis MA, Hughes $K$, Leckenby $N$, Perkins C, Lowey $H$. Nationa household survey of adverse childhood experiences and their relationship with resilience to health-harming behaviors in England. BMC Med. 2014;12:72.

3. Viola TW, Salum GA, Kluwe-Schiavon B, Sanvicente-Vieira B, Levandowski ML, Grassi-Oliveira R. The influence of geographical and economic factors in estimates of childhood abuse and neglect using the Childhood Trauma Questionnaire: a worldwide metaregression analysis. Child Abuse Negl. 2016;51:1-11.

4. Donoso MT, Ricas ]. [Parent's perspective on child rearing and corporal punishment]. Rev Saude Publica. 2009;43:78-84.

5. de Oliveira JR, Costa MC, Amaral MT, Santos CA, de Assis SG, do Nascimento OC. [Sexual violence and co-occurrences suffered by children and adolescents: study of incidents over a decade]. Cien Saude Colet. 2014;19:759-71.

6. Grassi-Oliveira R, Stein LM, Pezzi JC. [Translation and content validation of the Childhood Trauma Questionnaire into Portuguese language]. Rev Saude Publica. 2006;40:249-55.

7. Grassi-Oliveira R, Cogo-Moreira H, Salum GA, Brietzke E, Viola TW, Manfro GG, et al. Childhood Trauma Questionnaire (CTQ) in Brazilian samples of different age groups: findings from confirmatory factor analysis. PLoS One. 2014;9:e87118.

8. World Health Organization (WHO). Preventing child maltreatment: a guide to taking action and generating evidence. Geneva: WHO; 2006. p. 90

9. United Nations (UN). General Assembly: Rights of the child. 2006, p. 34. [cited 2016 Apr 26]. http://www.unicef.org/violencestudy/ reports/SG_violencestudy_en.pdf

10. Runyan $\mathrm{DK}$, Dunne $\mathrm{MP}$, Zolotor $\mathrm{AJ}$. Introduction to the development of the ISPCAN child abuse screening tools. Child Abuse Negl. 2009;33:842-5.

11. Zolotor AJ, Runyan DK, Dunne MP, Jain D, Peturs HR, Ramirez $C$, et al. ISPCAN Child Abuse Screening Tool Children's Version (ICAST-C): instrument development and multi-national pilot testing. Child Abuse Negl. 2009;33:833-41.

12. Dunne MP, Zolotor AJ, Runyan DK, Andreva-Miller I, Choo WY, Dunne SK, et al. ISPCAN Child Abuse Screening Tools Retrospective version (ICAST-R): Delphi study and field testing in seven countries. Child Abuse Negl. 2009;33:815-25.

13. Runyan DK, Dunne MP, Zolotor AJ, Madrid B, Jain D, Gerbaka B, et al. The development and piloting of the ISPCAN Child Abuse Screening Tool-Parent version (ICAST-P). Child Abuse Negl. 2009;33:826-32. 
14. International Society for the Prevention of Child Abuse and Neglect (ISPCAN). ISPCAN Child Abuse Screening Tools (ICAST) Questionnaires and Guides available for UN Study on Violence against Children [Internet]. 2015 [cited 2016 Apr 26]. http:// www.ispcan.org/?page =ICAST

15. Beaton DE, Bombardier C, Guillemin F, Ferraz MB. Guidelines for the process of cross-cultural adaptation of self-report measures. Spine (Phila Pa 1976). 2000;25:3186-91.

16. Guillemin F, Bombardier C, Beaton D. Cross-cultural adaptation of health-related quality of life measures: literature review and proposed guidelines. J Clin Epidemiol. 1993;46:1417-32.

17. Hasselmann MH, Reichenheim ME. [Cross-cultural adaptation of the Portuguese version of the Conflict Tactics Scales Form R (CTS1) used to assess marital violence: semantic and measurement equivalence]. Cad Saude Publica. 2003;19:1083-93.

\section{Correspondence:}

Rodrigo Grassi-Oliveira

Avenida Ipiranga, 6681 , prédio 11 , sala 928 , Partenon

90619-900 - Porto Alegre, RS - Brazil

Tel.: +55 (51) 3320.3633 - Ramal 7740

E-mail: rodrigo.grassi@pucrs.br 